\title{
Spectral, HR-XRD, Thermal and Dielectric Studies on Mixed Solvent Grown DAST Single Crystals
}

\section{Krishna Kumar}

\begin{abstract}
The highly meritorious single crystal ofDAST (4-N,N-dimethylamino-4'- $N$ '-methylstilbazolium tosylate) was grown through solution growth technique. The grown single crystals crystalline perfection analysis was made by HRXRD studies. The dielectric constant ( $\varepsilon$ ), dielectric loss ( $\left.\varepsilon^{\prime \prime}\right)$ of DAST crystal was studied at different temperatures. The thermal decomposition stages were examined by using thermogram of DAST crystal and also it was giving best description of kinetic changes. Dielectric character was studied to the DAST crystal. The dielectric character of DAST was too analyzed.
\end{abstract}

Keywords: Solution growth, HRXRD, Thermo-gravimetric analysis, Dielectric studies.

\section{INTRODUCTION}

The detection of terahertz $(\mathrm{THz})$ frequencies in organic compounds research is increasing in recent past years and large amount of research has been carried out. 4-N,N-Dimethylamino-4'N'-methylstilbazolium tosylate (DAST) crystals was studied by many researchers for nonlinear optics (NLO) applications and found its $\mathrm{THz}$ emission range [1-4]. The finding of $\mathrm{THz}$ frequency producing materials with high signal with noise ratio demands, its role in NLO and THz applications, because of owing its excellent NLO susceptibilities $(\chi 111=1230 \mathrm{pm} / \mathrm{V}$ at $800 \mathrm{~nm})$ with electro-optic coefficients $(\mathrm{r} 111=77 \mathrm{pm} / \mathrm{V}$ at $800 \mathrm{~nm}$ ) [5-6]. But, in the required size and specific orientation grown DAST for specific technology needs are still complicated, but it has unavoidable characteristics. DAST crystal growth in different solvents are undertaken to grow larger sized crystal without any grain boundaries and it was examined by HRXRD studies. The thermal degradation kinetics may help better knowledge and planning to grow best quality DAST crystals [7-8]. Studies on kinetics mechanism of reactions involves in solid compounds are complicated and difficult task for finding its results. It includes a re-construction of formation crystal lattice, and growth of new crystallization nuclei, etc. Thermogravimetric analysis (TGA), differential thermal analysis (DTA) are prettyvaluable, since they deliverunfailingevidence on physico-chemical parameters. The aim of this paper is to

Revised Manuscript Received on December 15, 2019

* Correspondence Author

M. Krishna Kumar, Department of Physics, Kalasalingam Academy of Research and Education, Krishnankoil-626 126, India. Email: m.krishnakumar@klu.ac. assess the crystal defects, kinetic models of DAST thermal degradation, estimation of dielectric loss and dielectric constant results are reported.

\section{EXPERIMENTAL}

\section{A.Crystal growth}

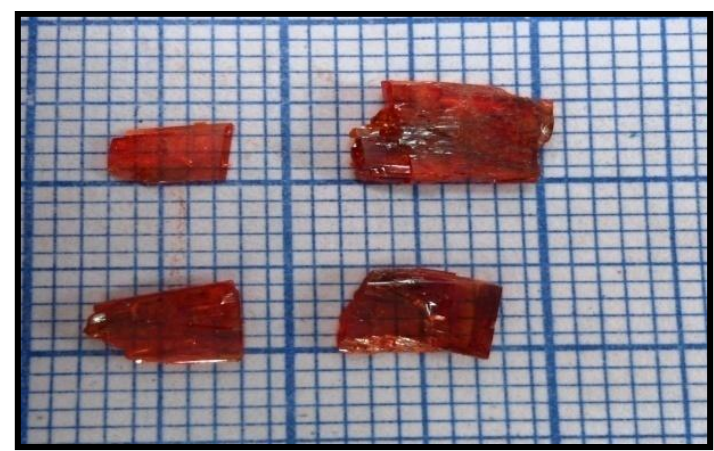

Fig.1a. As grown DAST crystal in methanol

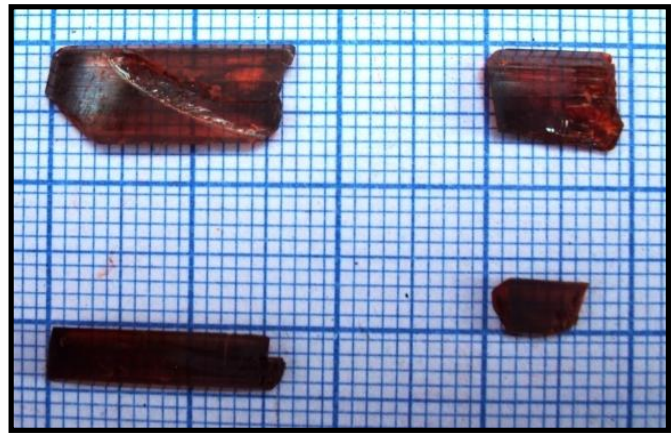

Fig.1b. As grown DAST crystal in methanol and water

The twin free DAST single crystals were grown by slope nucleation method (Fig.1a, and Fig.1b). The slope nucleation crystal growth method, facilitates controlled nucleation coupled with isothermal solvent evaporation procedure under lower supersaturation. DAST salt was prepared by the procedure as in our past research reports [9-10]. DAST salts were liquified in methanol and saturated solution was achieved and kept at constant temperature $(40 \mathrm{oC})$. Similarly, another saturated DAST solution was prepared with mixed solvent of methanol and millipore water $(9: 1)$ ratio. These two solutions were allowed to evaporate slowly at $40 \mathrm{oC}$.

Published By:

Blue Eyes Intelligence Engineering \& Sciences Publication

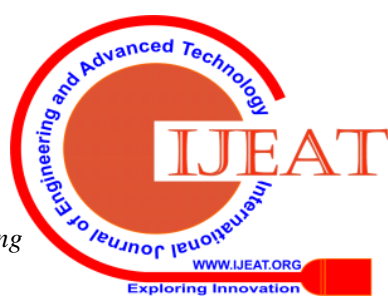




\section{Spectral, HR-XRD, Thermal and Dielectric Studies on Mixed Solvent Grown DAST Single Crystals}

The spontaneously nucleated DAST single crystals were obtained after 5 days $(4 \times 3 \times 3 \mathrm{~mm} 3$ and $16 \times 7 \times 2 \mathrm{~mm} 3)$ from both solutions respectively. It has been observed that, addition of water into growth solution is increasing its growth size and growth rate in all dimensions. The solubility was higher in mixed solvent and less in methanol alone. Also in the methanol and millipore water evaporation rate also slower than methanol. It was the driving force for the growth of larger sized crystal than pure methanol. The harvested crystals were kept safely for further studies. The mixed solvent grown crystal was used for the characterization.

\section{RESULTS AND DISCUSSION}

\section{A.FTIR studies}

The FTIR spectrum was noted using "JASCO FTIR 410 SPECTROMETER" and recorded spectrum are depicted in Fig.2. The absorbance and transmittance vibrations of the N, N-dimethylamino-4'N'-methyl stilbazolium tosylate (DAST) crystals grownup by methanol and water were recorded in the range of $400-4000 \mathrm{~cm}-1$ by using $\mathrm{KBr}$ pellet technique. The observed frequencies vibrations are assigned to the corresponding vibrational bonds and molecules. The assignments are confirming the synthesized compound of DAST perfectly. The peak $3430 \mathrm{~cm}^{-1}$ matches to the $\mathrm{O}-\mathrm{H}$ bonding in the DAST material. $3033 \mathrm{~cm}^{-1}$ reveals the vibrations owing to the aromatic $\mathrm{C}-\mathrm{H}$ stretching. The Alkyl $\mathrm{C}-\mathrm{H}$ stretching peaks available at $2922 \mathrm{~cm}^{-1}$. The $\mathrm{C}=\mathrm{C}$ bonding vibrations are present at $1645 \mathrm{~cm}^{-1}$. The peaks of $1584 \mathrm{~cm}^{-1}$ and $1526 \mathrm{~cm}^{-1}$ are arising due to the skeletal vibrations of aromatic rings. The $\mathrm{CH}_{2}$ bending and $\mathrm{C}-\mathrm{S}$ stretching vibrations present in the recorded spectrum at 1369 $\mathrm{cm}^{-1}$.

The vibrations of sulphonate group bonding $\mathrm{S}=\mathrm{O}$ arise at $1164 \mathrm{~cm}^{-1}$ and $1180 \mathrm{~cm}^{-1}$. The important vibrations of ortho, para positions of the aromatic ring $(1,4)$ was found at 824 $\mathrm{cm}^{-1}$. The olefinic double bond substitution peaks were present at $684 \mathrm{~cm}^{-1}$.

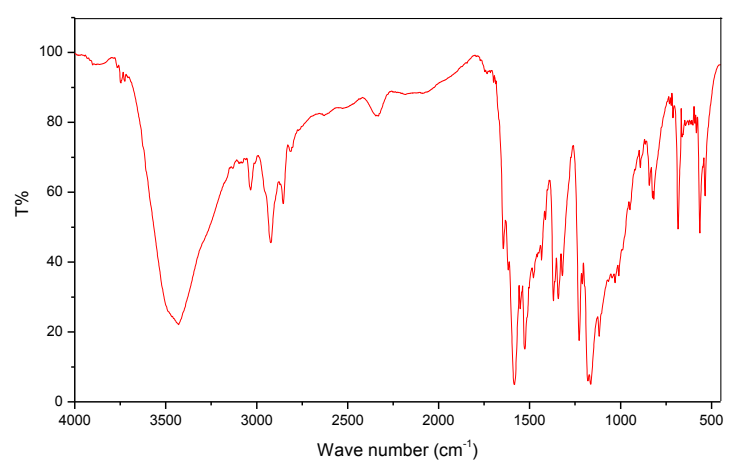

Fig.2. FT-IR spectra of DAST crystal grown in methanol and water

\section{B. HRXRD study}

The crystalline rightness of grown DAST single crystal was studied by HRXRD (high resolution X-ray diffraction) study by commissioning a multi-crystal X-ray diffractometer [11]. The separation of the X-ray beam evolving from a fine focus X-ray tube (X-ray generator (Philips, $2 \mathrm{~kW}$ with Mo) is first concentrated by a long collimator fitted with a pair of fine slit assemblies. The foreshortening angle of the evolving beam from the target was set aside at 3 degrees instead of the typical value of 6 degrees though the intensity is projected to be lesser in view of having healthier resolution. Molybdenum (Mo) target was preferred instead of frequently used $\mathrm{Cu}$ as the wavelength of MoK $\alpha 1(0.70926)$ is less than half of that of $\mathrm{CuK \alpha} \alpha(1.54056 \AA)$ ) and henceforth the predictable resolution for HRXRD experiments is added. The omega scan is very suitable to record the rapid range order scattering triggered by the defects or by the scattering from local Bragg diffractions from agglomerated point defects or owed to low angle and very small angle structural grain margins [12].

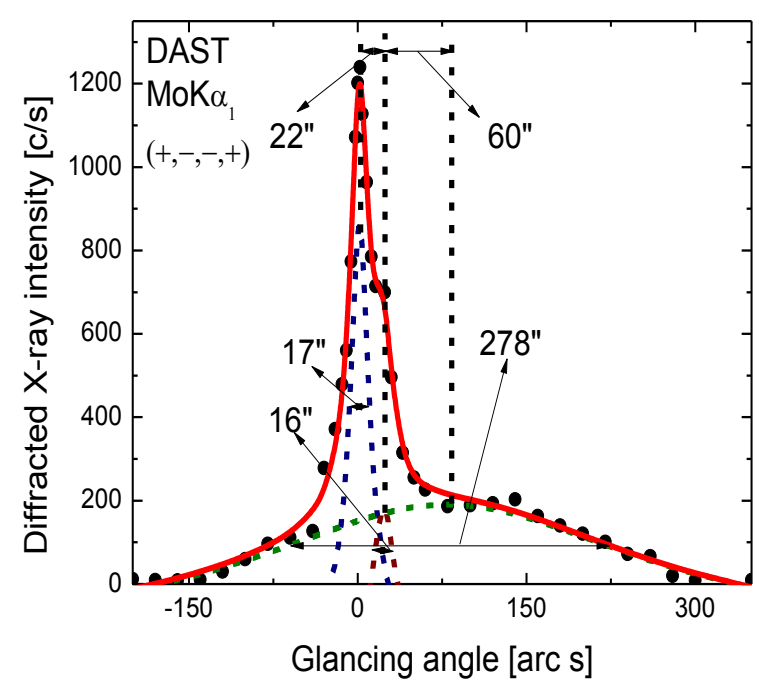

Fig.3 HRXRD curve on (001) plane of DAST crystal

In the circumstance of 2theta-theta or 2theta-omega scan, the experimentally attained RC comprises the evidence about a solo grain for which we bring into line for the scan and stunning curve is expected to be very high-pitched as the sensor with narrow slit will not receive diffracted intensities from the other grains which are mis-oriented to the grain under study or the long-winded scattering from point defects and their collections. Whereas in the relatively simple omega scan, with sufficiently wide slit for the detector, if the crystal contains structural grain boundaries, we got entire the peaks in the rocking curve (RC). On the other hand, in omega scan if we get a single piercing peak, it truly confirms that, the sample crystal has a single domain with sole orientation [13]. The well-collimated and monochromatic MoKal beam received from the three monochromator of Si crystals set in dispersive $(+,-,-)$ configuration. DAST crystal specimen was affiliated in the $(+,-,-,+)$ arrangement. The specimen was rotated perpendicular to the plane of diffraction around the vertical axis by smallest angular interval of 0.4 arc sec. The diffraction curves (DC) were logged by altering the glancing angle around the Bragg diffraction peak position $\theta_{\mathrm{B}}$. The DC was recorded by the $\alpha$ scan methods, wherein the detector was kept at the same angular position 20B [14]. Non-crystallized solute atoms remained on the surface of the crystal was removed before recording diffraction peaks [15] and also surface planarity was ensured.

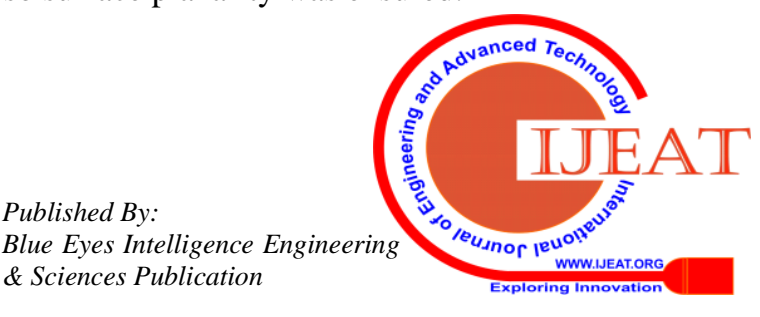


Fig.3 is the high resolution X-ray rocking/diffraction curve recorded in (001) planes of DST crystal using MoK $\alpha 1$ radiation.I show that, curve is not a single peak and it is clear that thereare two additional peaks which are 22 and 60 arc sec away from the main peak. The extra peaks correspond to the two very low angle grain boundaries of internal structure. [16]. The angular separation between the two side peaks has the tilt angle of $\alpha$ such as 22 and 60 arc s. The full width half maximum (FWHM) of main peak and the boundaries are respectively 17 and 16 and 278 arc s. The low FWHM values of DAST crystal and very low angle declares that, DAST crystal perfection is good.In some regions, because of broad peak (278 arc s) and low peak intensity indicates that crystal is not perfect and contains mosaic blocks. It is due to structural defects, probably due to strains produced in crystal may be from impurities or dopants [16-17]. This perfection with non-center of symmetry yields highest NLO properties to the DAST crystals.

\section{C.TGA/DTA analysis}

The thermal stability of a solid materials must be evaluated for its withstanding ability for different application. In our case DAST crystals are used I nonlinear optical applications measurements, which hold high heat production like LASER.It is important to study thermal degradation of DAST and carried for it in a flow of synthetic air at a rate of $25 \mathrm{~cm}^{3}$ min-1 under non-isothermal conditions by using an instrument Nietzsch STA 449 F3 Jupiter. The DAST sample weight of $7 \mathrm{mg}$ mass were taken and loaded without pressing into an open $6 \mathrm{~mm}$ diameter and $3 \mathrm{~mm}$ high platinum crucible, without using of a standard reference material. Temperature was varied out in $3,6,9,12,15$ and $18^{\circ} \mathrm{C}$ min upto $800^{\circ} \mathrm{C}$ (Fig.4). Results of TG, DTG and DSC curves were recorded simultaneously with $0.1 \mathrm{mg}$ sensitivity. The peak at $261^{\circ} \mathrm{C}$ is the decomposition starting point of DAST and afterwards in different stages the compound will get loose its entire structure.

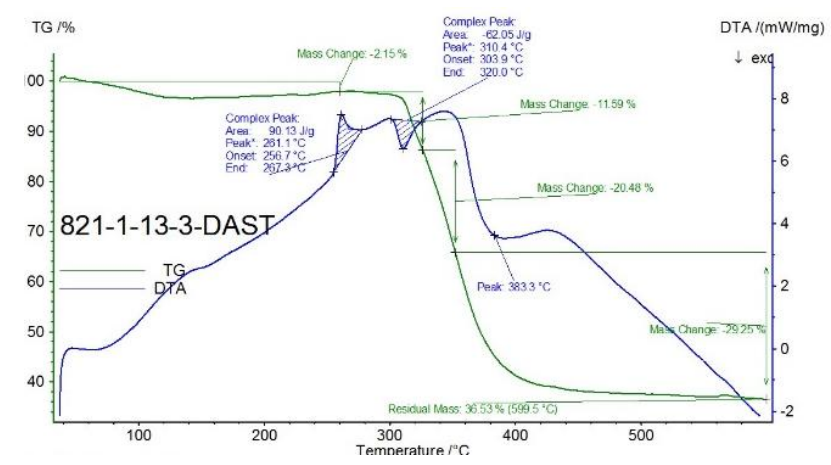

Fig.4TGA/DTA of DAST crystal

\section{D.Dielectric studies}

The single crystal of DAST of $5.76 \times 4.29 \times 0.95 \mathrm{~mm} 3$ were subjected to dielectric studies at temperatures of $40,60,80$, 100, 120 degree Celsius. The opposite faces of (001) plane of DAST crystal was applied by silver past for smooth electric conduction and placed between the copper electrodes. This is the model of simple capacitor, this capacitance of the sample was measured by varying the frequency from $50 \mathrm{~Hz}$ to $5 \mathrm{MHz}$ using LCR HITESTER. The dielectric constant is evaluated by using the relation,

$$
\varepsilon_{\mathrm{r}}=\mathrm{Ct} / \varepsilon_{\mathrm{o}} \mathrm{A}
$$

Where $\varepsilon_{0}$ is the permittivity of free space, $\varepsilon_{\mathrm{r}}$ is the relative permittivity, $\mathrm{C}$ is the capacitance of the material, $\mathrm{T}$ is the thickness of the material, A is the area of the cross section.

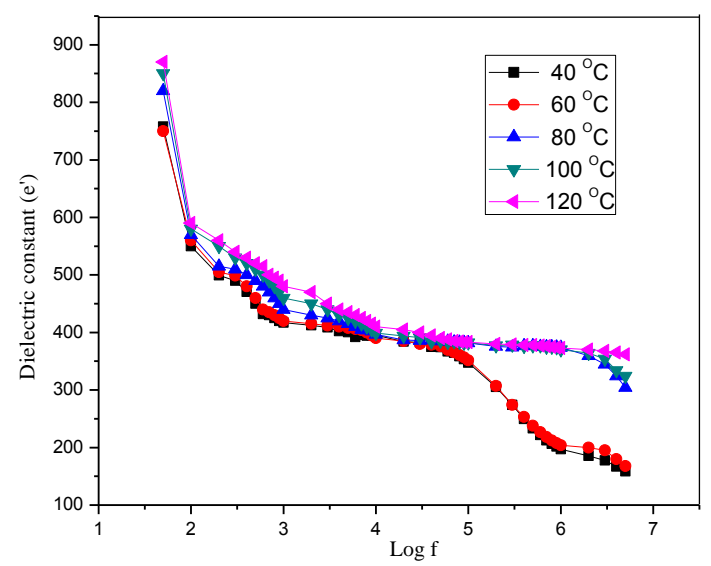

Fig.5 Plot of dielectric constant vs. frequency of DAST crystal

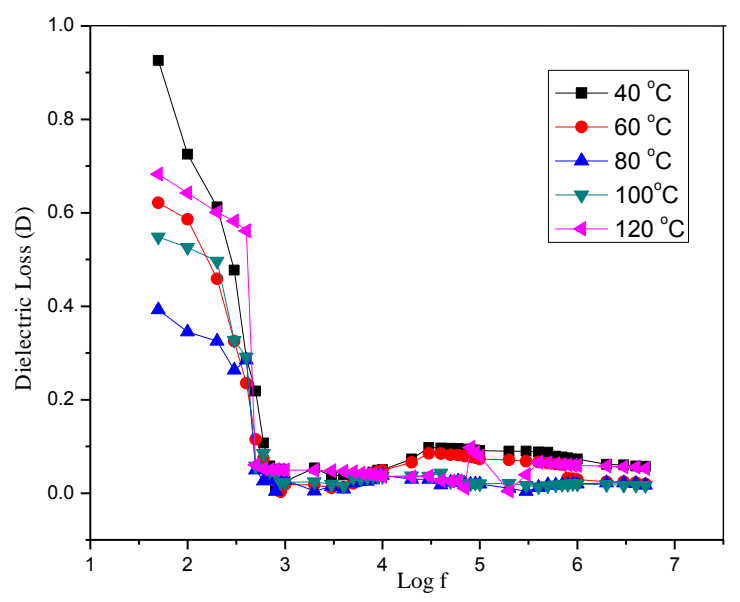

Fig.6 Plot of dielectric loss vs. log frequency of DAST crystal

The capacitance of the DAST material was measured by applying various frequencies by applying electric field. Dielectric constant and dielectric loss have been calculated from observed values with above said relation. Dielectric constant and dielectric loss are inversely proportional to the applied frequency. In the lower frequency dielectric constant showed higher value, when increasing the frequency, the dielectric constant decreasing and it is constant at higher frequencies (Fig.5). The similar dielectric loss calculation was made and drawn plot is given in Fig.6. It also, shows the increase of loss at lower applied frequencies.

\section{CONCLUSSION}

4-N,N-Dimethylamino-4'N'-methylstilbazolium tosylate (DAST) single crystal was grown in pure methanol and water mixed with methanol.

Published By:

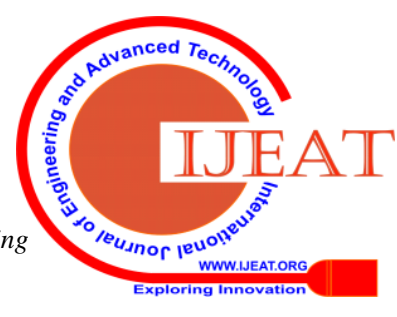




\section{Spectral, HR-XRD, Thermal and Dielectric Studies on Mixed Solvent Grown DAST Single Crystals}

The increased size of DAST was grown from methanol and water mixed solvent. The growth from mixed solvent was attempted and characterized. The FT-IR studies were confirming it was DAST compound. The HRXRD study revealed that, low angle boundaries were present at 22 and 60 arc s from their adjoining regions. The full width at half maximum of the main peak and the boundaries are respectively 17 and 16 and 278 arc s. The melting point of DAST crystal grown in methanol and water is found to be $261^{\circ} \mathrm{C}$. Dielectric constant and dielectric loss were measured for DAST crystals and the results were reported.

\section{REFERENCES}

1. H. Adachi, T. Taniuchi, M. Yoshimura, S. Brahadeeswaran, T. Higo, M. Takagi, Y. Mori, T. Sasaki, H. Nakanish, Jpn. J. Appl. Phys, Vol.43, pp. 1121-1123 (2004).

2. Z. Yang, M. Jazbinsek, B. Ruiz, S. Aravazhi, V. Gramlich, P. Gunter, Chem. Mater. Vol.19, pp.3512-3518 (2007).

3. M. Krishna Kumar, S. Sudhahar, P. Pandi, G. Bhagavannarayana, R. Mohan Kumar, Opt. Mat. Vol.36, 988-995 (2014).

4. M. Joffre, D. Yaron, R.J. Silbey, J. Zyss, J. Chem. Phys. Vol. 97, pp. 5607-5615 (1992).

5. A. Schneider, M. Neis, M. Stillhart, B. Ruiz, R. Khan, P. Gunter, J. Opt. Soc. Am. B, Vol.23, pp. 1822-1835 (2006).

6. J. Zyss, Nonlinear Opt. Vol.1, pp. 3-18 (1991).

7. S.R. Marder, J.W. Perry, C.P. Yakymyshyn, Chem. Mater. Vol.6, pp. 1137-1147 (1994).

8. S.R. Marder, J.W. Perry, B.G. Tiemann, Chem. Mater. Vol.2, pp. 685-690 (1990).

9. S.R. Marder, B. Kippelen, A.K. Jen, N. Peyghambarian, Nature, Vol.388, pp.845-851 (1997).

10. Z. Yang, S. Aravazhi, A. Schneider, P. Seiler, M. Jazbinsek, P. Gunter, Adv. Funct. Mater. Vol.15, pp. 1072-1076 (2005).

11. Krishan Lal and G. Bhagavannarayana, J. Appl. Cryst. Vol.22, pp. 209-215 (1989).

12. G. Bhagavannarayana, S.K. Kushwaha, J Appl. Cryst. Vol43, pp. 154-162 (2010).

13. KrishanLal and G. Bhagavannarayana, J. Appl. Cryst. Vol.22, pp.209-215 (1989).

14. G. Bhagavannarayana and S. K. Kushwaha, J. Appl. Cryst. Vol.43, pp.154-162 (2010).

15. G. Bhagavannarayana, S. Parthiban and Subbiah Meenakshisundaram, J. Appl. Cryst.Vol.39, pp.784-790 (2006).

16. G. Bhagavannarayana, P. Rajesh, and P. Ramasamy, J. Appl. Cryst. Vol.43, pp.1372-1376 (2010).

17. G. Bhagavannarayana, B. Riscob and Mohd. Shakir,Materials Chemistry and Physics Vol.126, pp. 20-23 (2011). 